\title{
EPLIN: a fundamental actin regulator in cancer metastasis?
}

\author{
Ross J. Collins ${ }^{1}$ - Wen G. Jiang ${ }^{1} \cdot$ Rachel Hargest $^{1}$ • Malcolm D. Mason ${ }^{2}$ • \\ Andrew J. Sanders ${ }^{1}$
}

Published online: 9 September 2015

(C) The Author(s) 2015. This article is published with open access at Springerlink.com

\begin{abstract}
Treatment of malignant disease is of paramount importance in modern medicine. In 2012, it was estimated that 162,000 people died from cancer in the UK which illustrates a fundamental problem. Traditional treatments for cancer have various drawbacks, and this creates a considerable need for specific, molecular targets to overcome cancer spread. Epithelial protein lost in neoplasm (EPLIN) is an actin-associated molecule which has been implicated in the development and progression of various cancers including breast, prostate, oesophageal and lung where EPLIN expression is frequently lost as the cancer progresses. EPLIN is important in the regulation of actin dynamics and has multiple associations at epithelial cells junctions. Thus, EPLIN loss in cancer may have significant effects on cancer cell migration and invasion, increasing metastatic potential. Overexpression of EPLIN has proved to be an effective tool for manipulating cancerous traits such as reducing cell growth and cell motility and rendering cells less invasive illustrating the therapeutic potential of EPLIN. Here, we review the current state of knowledge of EPLIN, highlighting EPLIN involvement in regulating cytoskeletal dynamics, signalling pathways and implications in cancer and metastasis.
\end{abstract}

Keywords EPLIN $\cdot$ Cancer $\cdot$ Metastasis $\cdot$ Actin

Ross J. Collins

Collinsr6@cardiff.ac.uk

1 Cardiff China Medical Research Collaborative (CCMRC), Cardiff University School of Medicine, Henry Wellcome Building, Heath Park, Cardiff CF14 4XN, UK

2 Department of Clinical Oncology, Cardiff University School of Medicine, Cardiff, UK

\section{Introduction}

The incidence of cancer is slowly rising and has become a global burden. A fundamental reason why cancer is such a problem is because of its ability to spread, invade surrounding tissue and potentially form secondary cancers at distinct sites around the body by metastasis. Cancer hallmarks include uncontrolled cell growth and evasion of cell death, and this ultimately can lead to tumour formation. According to the World Health Organisation (WHO), 8.2 million people died from cancer in 2012 worldwide [1]. In the UK alone, mortality rates reached 162,000 annual deaths [2]. This illustrates a considerable need for better treatment, diagnosis and management of the disease. Epithelial protein lost in neoplasm (EPLIN) is a molecule involved in regulation of the actin cytoskeleton and has been implicated in the development and progression of various cancer types, displaying frequent downregulation or loss in cancer, creating a potential for prognostic targeting and as a tumour suppressor. This current review discusses EPLIN's role in actin dynamics and in the pathophysiology of cancer development and progression.

\section{Epithelial protein lost in neoplasm}

EPLIN is a cytoskeletal, actin-binding protein encoded by the LIMA1 gene. EPLIN was initially identified in oral cancers for its differential expression between normal oral epithelial cells and human papilloma virus (HPV)-immortalised oral epithelial cells [3]. EPLIN exists as two distinct isoforms, a 600 amino acid EPLIN $\alpha$ isoform and a larger 759 amino acid EPLIN $\beta$ isoform, generated from an alternative pre-mRNA splicing event (see Fig. 1) [4]. The EPLIN $\alpha$ isoform has been implicated in the progression of various cancers, and this was initially recognised in oral cancer, breast, prostate and 
EPLIN- $\alpha$ Isoform - 600aa
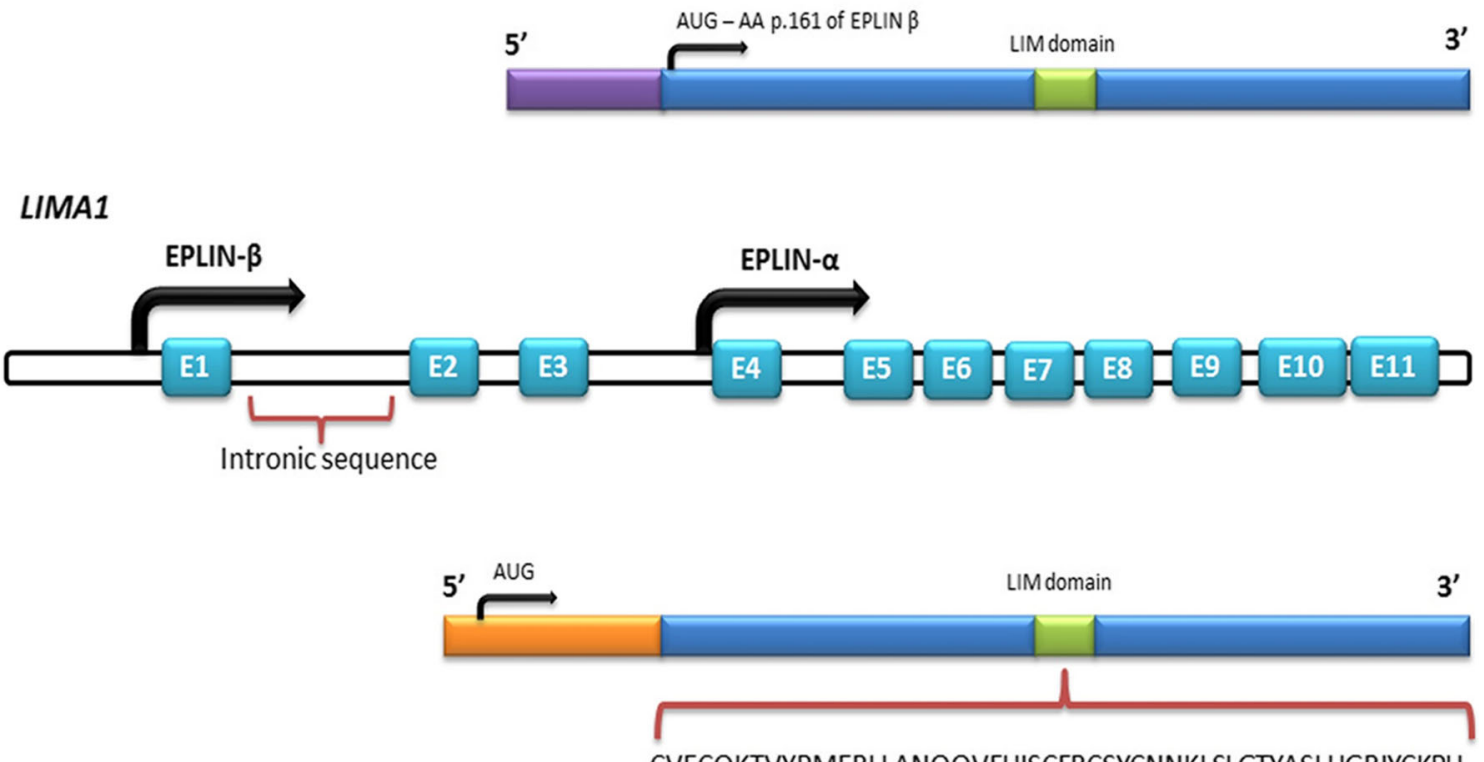

EPLIN- $\beta$ Isoform - 760aa

Fig. 1 Schematic diagram of the LIMAl genomic structure and EPLIN structural isoforms. The LIMA1 gene consists of 11 exons and ten introns. EPLIN $\alpha$ differs from EPLIN $\beta$ at the amino terminus where an additional

xenograft tumours where EPLIN expression was either downregulated or completely abolished [4]. The amino acid sequence of EPLIN is characterised by a single centrally located LIM domain which supposedly aids structural selfdimerisation and contains subdomains for zinc binding (see Fig. 2) [4, 5]. LIM-domain-containing proteins are frequently present in molecules responsible for cytoskeletal organisation, such as the focal adhesion phosphoprotein, paxillin [6]. EPLIN is important in the regulation of actin dynamics and aids actin filament bundle assembly, and the amino terminal of the EPLIN protein structure is essential for this localisation to the actin cytoskeleton [7]. The EPLIN genomic structure consists of 11 exons and ten introns, with exons 1-3 only present in EPLIN $\beta$ and EPLIN $\alpha$ utilising exons 4-11 of LIMA1 for transcription [5]. The EPLIN gene has two separate promoter regions; the EPLIN $\beta$ promoter is near the start of the gene in exon 1, whilst EPLIN $\alpha$ initiates $\sim 50 \mathrm{~kb}$ downstream near the end of intron 3, prior to exon 4 and at amino acid position 161 in the EPLIN $\beta$ protein (see Fig. 1) [5]. Sequence analysis has revealed that EPLIN is conserved across species with EPLIN $\alpha$ and EPLIN $\beta$ isoforms present in mouse, displaying 77 and $75 \%$ identity similarity for human EPLIN $\alpha$ and EPLIN $\beta$, respectively (see Fig. 3) [8]. A role for EPLIN has also been suggested in muscle development in pigs, where EPLIN displayed a temporal expression pattern with only the EPLIN $\alpha$ isoform present in developing skeletal muscle [9]. Since the discovery of EPLIN, our lab has shown that aberrant EPLIN expression is associated with the progression of
160 amino acids are present in EPLIN $\beta$. Shown below EPLIN $\beta$ is the 52amino acid centrally located LIM domain common to both EPLIN isoforms. Adapted from [4]

various cancer types including breast, oesophageal, pulmonary and prostate cancer. EPLIN $\alpha$ levels decrease as the cancer progresses and becomes more advanced, giving EPLIN $\alpha$ potential to provide prognostic value, and overexpression analysis suggests that EPLIN $\alpha$ is a putative tumour suppressor [10-14]. The described loss of EPLIN in cancer has functional implications on the actin cytoskeleton and may contribute to enhanced metastatic potential of cancer cells.

\subsection{The epithelial protein lost in neoplasm interactome: regulation in actin dynamics}

EPLIN has a number of functional partners (see Fig. 4/ Table 1), and the globular protein actin is central to the function of EPLIN. EPLIN has two functional actin-binding sites which flank the central LIM domain, and it is this binding capacity that engenders actin cross linking and actin filament bundle assembly [15]. A fibrillar pattern is displayed by both isoforms, and expression of EPLIN $\alpha$ enhances the size and number of actin filament stress fibres and can also inhibit membrane ruffling via the signalling GTPase, Rac1 [15]. EPLIN therefore directly interacts with actin which suggests a possible role for EPLIN in cell migration, adhesion and cell morphology. Actin is an abundant, multifunctional protein responsible for cell migration in eukaryotic cells. Actin is part of the cytoskeletal network which consists of microtubules, microfilaments and intermediate filaments which are vital for cellular functions. Actin exists as monomers (G-actin) and 


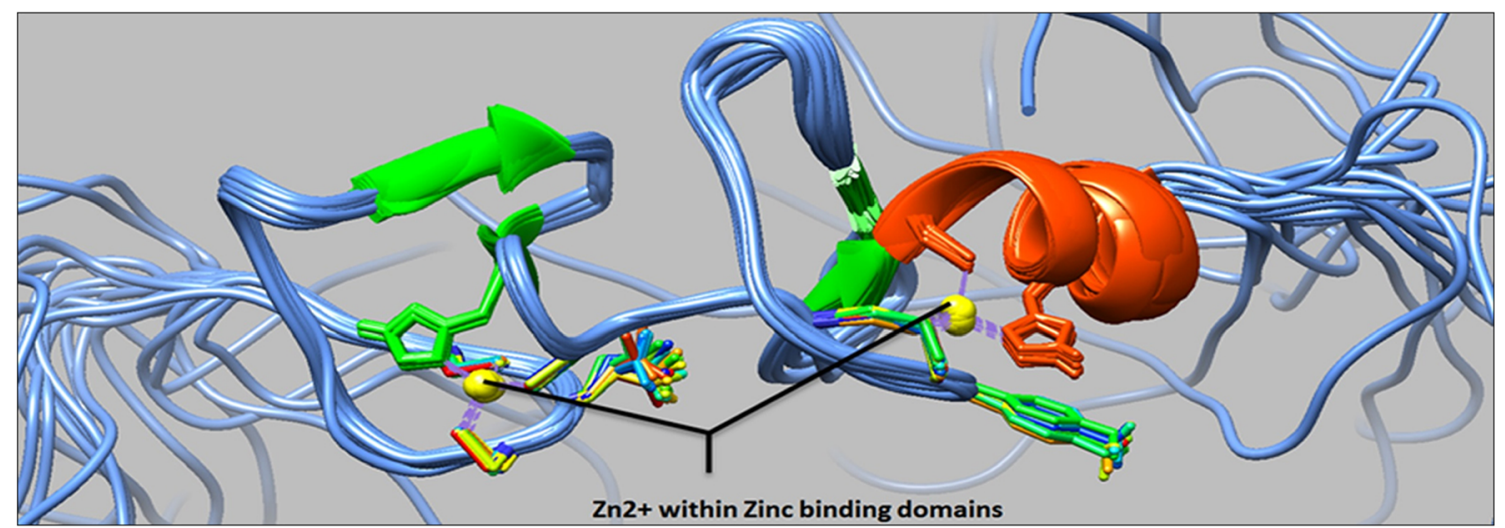

Fig. 2 Protein structure of EPLIN LIM domain (PDB ID=2D8Y). Protein structure of the EPLIN centrally located LIM domain. Zinc- binding domains depicted. This domain may aid self-dimerisation. Image generated using UCSF Chimera software filamentous polymers (F-actin) and is important for physiological functions including cell locomotion, cytokinesis, maintenance of cell shape and muscle contraction [24]. Transcription of the LIMA1 gene is suggested to be primarily controlled by monomeric $\mathrm{G}$ actin, with the actin-MAL-SRF signalling pathway regulating EPLIN production [25]. Maul et al. [15] illustrated that EPLIN $\alpha$ has three significant features: EPLIN $\alpha$ has at least one binding site for actin and can cross link and bundle actin filaments, EPLIN $\alpha$ stabilises actin filaments in vitro, and EPLIN $\alpha$ inhibits branching nucleation of actin filaments by the Arp 2/3 complex [15]. Therefore, this suggests that EPLIN may orchestrate actin filament dynamics by stabilising actin cytoskeletal networks [15]. Additionally, EPLIN has been shown to form part of an actin-remodelling complex composing of EPLIN, $\beta$-actin, $\gamma$-actin and gelsolin which co-localises at the plasma membrane to the tumour suppressor, phosphatase and tensin homolog (PTEN) [26]. PTEN is a well-established tumour suppressor molecule, so this asks the question of whether the interaction of PTEN and the actin-remodelling complex is itself an element suppressing the development of neoplastic tissue and whether any interruption of these complexes may promote cancer progression. Based on these findings, EPLIN accommodates actin to accomplish various actin-related cellular processes including cell motility and migration and cell junctional adhesion [17]. There is increasing evidence to suggest that EPLIN regulates actin structures in cooperation with the signal transduction adaptor protein, paxillin. When EPLIN is overexpressed, paxillin exhibits an increased staining pattern for both human endothelial cells line (HECV) and PC-3 cells [12, 13]. This colocalisation pattern is also observed in cultured human mesangial cells at focal adhesion sites, and coimmunoprecipitation results confirm an association between the two molecules [16]. EPLIN and paxillin may form a complex and potentially stabilise focal adhesions to co-ordinate actin dynamics in a complimentary manner. Given EPLIN's role in actin dynamics, it is strongly implicated in cellular processes including cell migration and invasion, and thus, downregulation or loss of EPLIN expression in cancer may likely affect the metastatic potential of cancer cells. Actin and EPLIN are located in epithelial cells at the adherens junction (AJ) and contribute to functional cellular adhesion between adjacent cells.

\subsection{The adherens junction}

The AJ is a type of anchoring junction found predominantly in epithelial cells, also referred to as the zonula adherens, which functionally link the actin cytoskeleton together in cells via

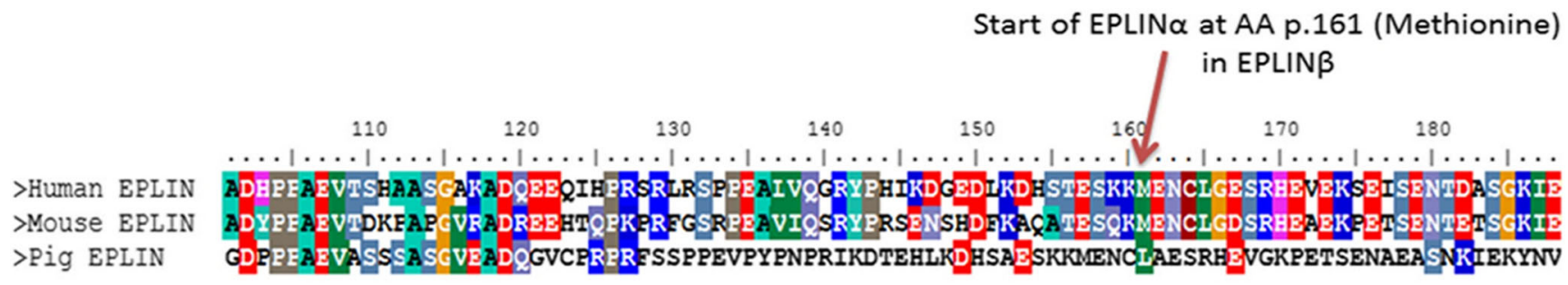

\section{EPLIN $\beta$ sequence (exons 1-3) upstream of EPLIN $\alpha$ start point}

Fig. 3 ClustalW protein alignment of human, mouse and pig EPLIN $\beta$. Areas of amino acids that are conserved across species are highlighted. The region shown is amino side of the EPLIN $\beta$ protein, where EPLIN $\alpha$ originates at amino acid (AA) p. 161. ClustalW generated using BioEdit Biological Sequence Alignment software 


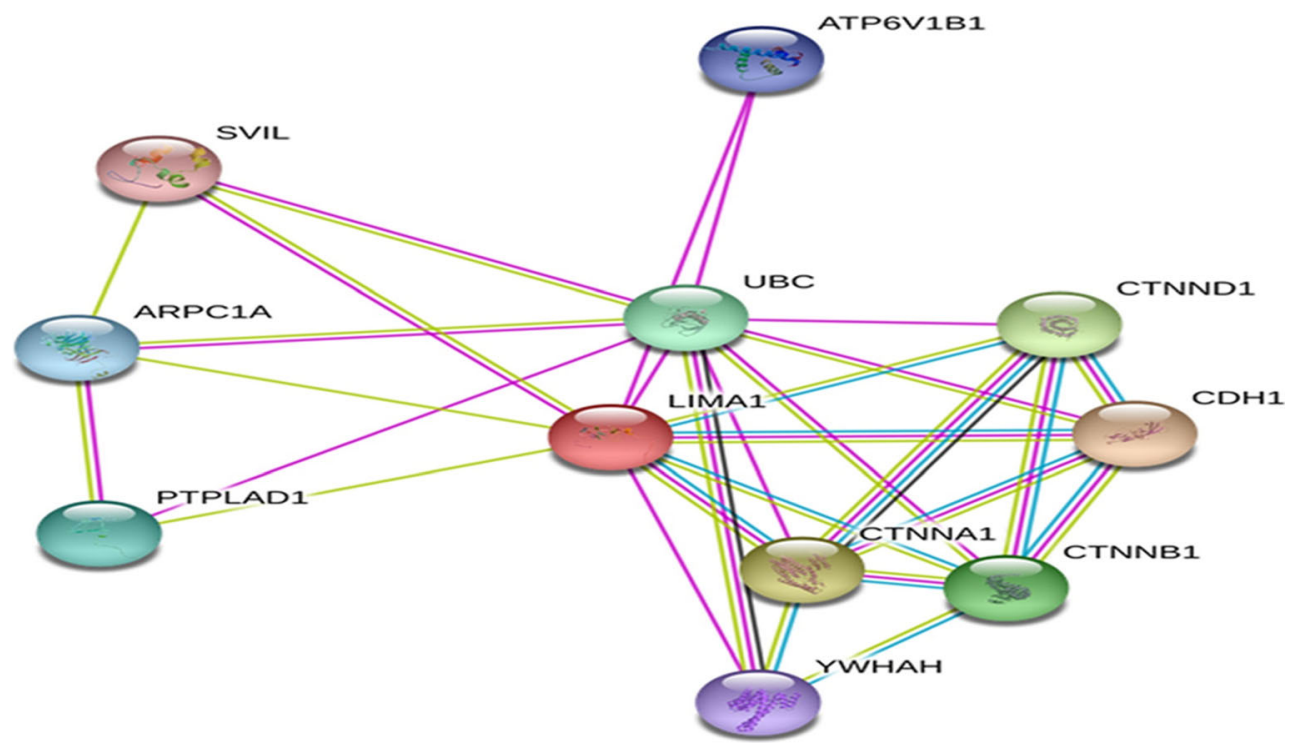

Fig. 4 EPLIN predicted functional partners. EPLIN (LIMA1) has various associations including cadherin and catenin molecules which contribute to cytoskeleton regulation. LIMAI LIM domain and actin binding 1; $C D H 1$ cadherin 1 ; CTNNA1 catenin- $\alpha 1 ; C D H 1$ cadherin $1 ;$ CTNND1 catenin- $\delta 1$; CTNNB1 catenin- $\beta 1 ; U B C$ ubiquitin $C$; PTPLAD1 protein tyrosine phosphatase-like A domain-containing 1 ; $A R P C 1 A$ actin-

linker molecules. EPLIN is an actin-binding protein, which functions to bundle actin filaments; therefore, EPLIN presence is required at $\mathrm{AJ}$ along with filamentous actin. AJ contains various protein complexes along with EPLIN, and these include cadherins, catenins and p120 catenins (see Fig. 5) [28]. Within the AJ, cadherins and catenins associate together to form the cadherin-catenin complex and EPLIN provides a direct physical link for this complex to the actin cytoskeleton related protein 2/3 complex, subunit $1 \mathrm{~A} ; A T P 6 V 1 B 1$ ATPase, $\mathrm{H}+$ transporting, lysosomal $56-58 \mathrm{kDa}, \mathrm{V} 1$ subunit $\mathrm{B} 1 ; Y W H A H$ tyrosine 3-monooxygenase/tryptophan 5-monooxygenase activation protein; SVIL supervillin. Image generated and extracted from online STRING database

[17]. The cadherin-catenin complex is composed of Ecadherin, $\beta$-catenin and $\alpha$-catenin, with E-cadherin positioned between adjacent cells and the catenins positioned in the cytoplasmic space of each cell [29]. E-cadherin binds directly to $\beta$-catenin which sequentially binds $\alpha$-catenin, generating the cadherin-catenin complex (see Fig. 5) [30]. $\alpha$-Catenin is a crucial player at the AJ and was initially recognised as responsible for providing the bridge between the cadherin-catenin

Table 1 EPLIN-associated molecules

EPLIN Biological significance and reference

References

association

Actin

Actin is an abundant protein important for cell migration. EPLIN has two actin-binding domains that flank the EPLIN LIM [15] domain. Pull-down assays revealed that EPLIN binds actin monomers, and this results in actin cross linking and actin filament bundle assembly.

May form a complex with EPLIN to co-ordinate actin dynamics. IHC of PCa tissue vs normal reveals that EPLIN overexpression influences paxillin expression and localisation. Co-localisation, co-precipitation and an in situ proximal 16] ligation assay revealed direct association between the two molecules in cultured human mesangial cells.

$\alpha$-Catenin $\quad$ Immunoprecipitation and GST pull-down assays reveal that EPLIN interacts with $\alpha$-catenin, forming a cadherin- $\beta$ catenin- $\alpha$-catenin-EPLIN complex.

Supervillin In vivo co-localisation studies and in vitro GST pull-down assays reveal that EPLIN interacts with the peripheral membrane protein, supervillain.

PINCH-1 Pull-down assays reveal that endogenous EPLIN co-immunoprecipitates with endogenous PINCH-1 in keratinocytes. [19]

ERK ERK phosphorylates EPLIN and decreases EPLIN affinity to F-actin promoting cell migration. Inhibition of ERK [10, 13, abolishes EPLIN expression and reduces tumour-suppressive ability of EPLIN.

DNp73 In melanoma cells, both EPLIN isoforms are inhibited by DNp73, and this drives a more invasive phenotype.

SATB2 EPLIN is differentially regulated by the DNA-binding protein, SATB2. SATB2 regulates the actin cytoskeleton via EPLIN association. When SATB2 is knocked out, osteosarcoma cells show reduced migration and are less invasive, and this is mediated by EPLIN.

Cav-1 EPLIN regulates the lipid raft tumour-suppressive protein, Cav-1. Co-immunoprecipitation and mass spectroscopy analysis revealed that EPLIN and Cav-1 bind to each other in normal and RasV12 cells. 


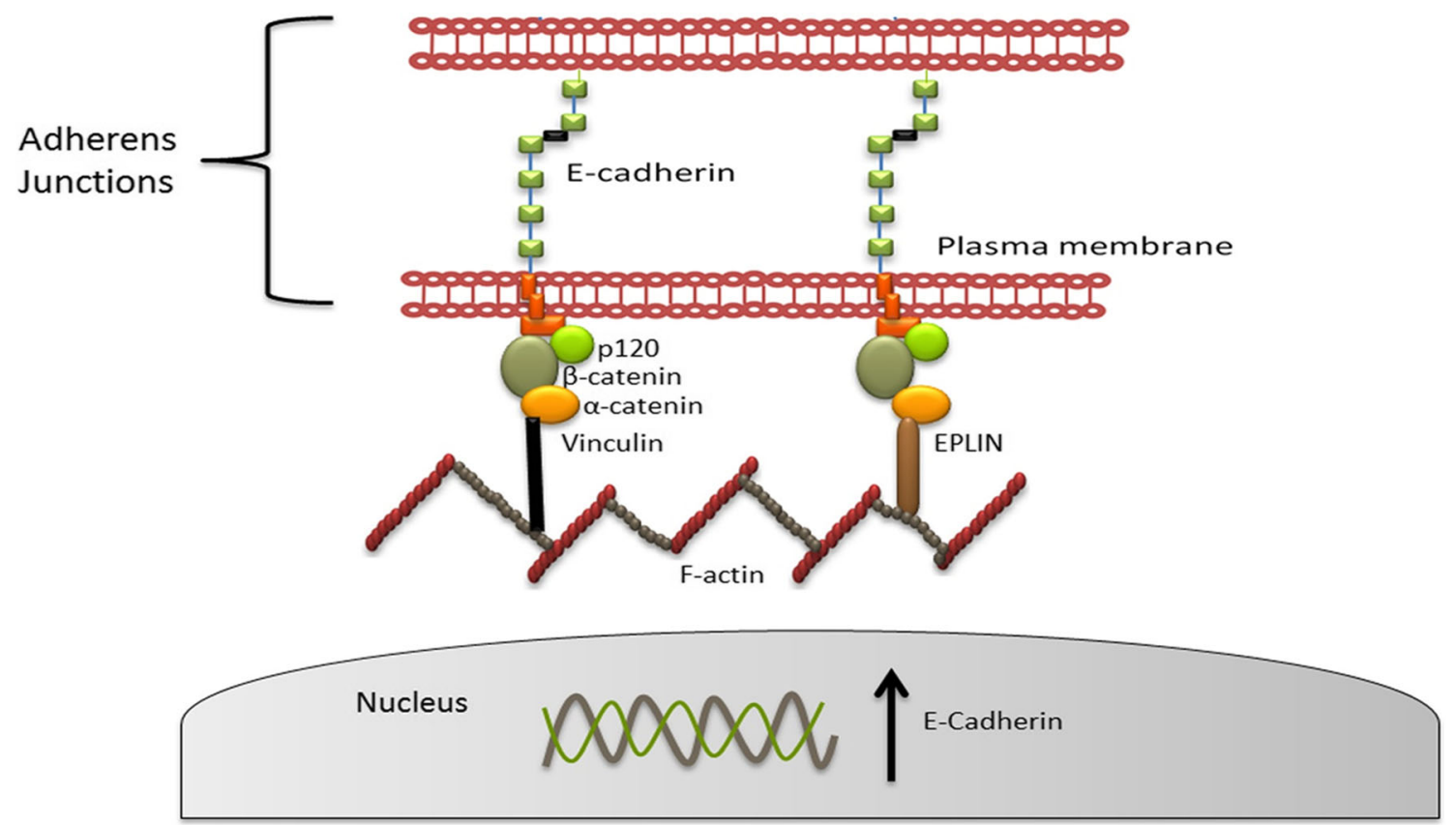

Fig. 5 Schematic representation of adherens junctions. The AJ between epithelial cells consists of various protein complexes to orchestrate actin cytoskeletal dynamics. The cadherin-catenin complex is associated with filamentous actin via EPLIN and/or vinculin which binds $\alpha$-catenin in the cytoplasm. Adapted from [27]

allows the recruitment of vinculin and ultimately promotes strengthening of inter-endothelial junctions [38]. The authors propose a role for EPLIN in tension dissemination at the endothelial AJ in a mechanosensory mechanism [38]. The machinery from actomyosin exerts tension through EPLIN, which causes $\alpha$-catenin to adopt a more accessible conformation, revealing a vinculin-binding site and allowing vinculin recruitment and actin association at endothelial cell-cell AJ [39]. This mechanotransduction mechanism consisting of EPLIN and $\alpha$-catenin suggests that the endothelial AJ is regulated in a spatial and temporal fashion [39]. Finally, EPLIN also appears to be important for attachment to F-actin in endothelial cells; when EPLIN expression is downregulated, the organisation of F-actin is considerably disrupted, leading to multiple holes in the actin cytoskeleton [38]. Altogether, this suggests that the AJs of epithelial and endothelial cells are orchestrated by various actin-binding, $\alpha$-catenin-associated molecules and are dynamically regulated, with EPLIN having a critical role in cell adhesion, creating further implications of EPLIN loss in cancer.

\subsection{Epithelial protein lost in neoplasm—a key player in cell division?}

Cell division is the splitting of one cell into two, where biological information is passed onto daughter cells. For this process to successfully occur, various proteins need to functionally regulate the division and these include Rho GTPases, cyclin-dependant kinases, integrins, cdc42, focal adhesion 
kinases, myosin and the globular protein actin [40]. With this in mind, an actin-binding protein like EPLIN may potentially have a regulatory role in cell division. This has been recently shown using HeLa cells where EPLIN depletion resulted in large numbers of multinucleated cells, signifying cytokinesis failure during cell division [41]. In successful mitotic division, actin and myosin II accumulate at the cleavage furrow during cytokinesis and EPLIN loss compromised each protein's ability to efficiently do this [41]. EPLIN appears to be important for the accumulation of other mitotic regulatory proteins including the GTPases RhoA and cdc42, where EPLIN depletion resulted in either a significantly reduced concentration of RhoA or a misplaced location of cdc 42 at the cleavage furrow [41]. EPLIN aids this successful cell division in conjunction with a number of regulatory proteins including supervillin and the oncogenic kinesin, KIF14, suggesting a complex network of regulatory proteins at the cleavage furrow [18]. Altogether, this suggests that EPLIN may have an integral role in cytokinesis and loss may lead to aneuploidy and genomic instability of daughter cells [41]. Therefore, EPLIN is crucial to coordinate actin and myosin dynamics throughout cell division and loss in cancer cells could have downstream effects on successful cytokinesis, increasing their tendency to form a cancer [41].

\subsection{Post-translational modification}

Extracellular signal-regulated kinase (ERK) is a member of the mitogen-activated protein kinase (MAPK) family and is important in the regulation of actin organisation by phosphorylating various proteins including paxillin, focal adhesion kinase (FAK) and other protein kinases and nuclear transcription factors to co-ordinate cellular processes $[42,43]$. ERK is implicated in cellular events including cell migration and may facilitate this by phosphorylation of actin-bundling proteins like EPLIN [20]. The protein structure of EPLIN has multiple putative phosphorylation sites (see Fig. 6), and it has been shown that ERK phosphorylates EPLIN at Ser360, Ser602 and Ser692 in vitro and in vivo [20]. Phosphorylation at the carboxy terminal of EPLIN decreases affinity to F-actin and thus provokes a reorganisation of the actin cytoskeleton, enhancing cell migration [20]. This implicates ERK in actin organisation and cell motility with EPLIN being a critical substrate for phosphorylation [20]. A recent study by Zhang et al. [44] illustrated that this ERK-mediated phosphorylation of EPLIN is itself regulated by epidermal growth factor (EGF) and revealed how targeting this signalling cascade can be manipulated to reduce epithelial-mesenchymal transition (EMT) and, thus, prostate cancer invasiveness [44]. ERK also plays a role in targeting EPLIN to focal adhesions and effects the interaction with paxillin; activation of the MEK-ERK pathway both reduced localisation of EPLIN to sites of focal adhesions and abolished paxillin interaction [16]. These data suggest that ERK is functionally related to EPLIN and provides a critical regulatory role for appropriate actin dynamics and may have implications in cancer progression.

\section{The role of epithelial protein lost in neoplasm in cancer}

Cancer progression involves various cellular, morphological and molecular alterations which result in a transformed cellular phenotype, ultimately having the potential to invade surrounding tissue and disseminate throughout the body. Cancer treatment options remain largely unspecific and create various undesired side effects. Therefore, elucidating a molecular target for treating cancer, in addition to understanding the mechanism of cancer development, is crucial. EPLIN first received attention for its involvement in cancer in 1999 where EPLIN downregulation was described in various cancer cell lines [4]. Altogether, low levels of EPLIN transcript were found in $8 / 8$ oral cancer cell lines, $5 / 6$ breast cancer cell lines and $4 / 4$ prostate cancer cell lines [4]. Using PC-3 and DU-145 prostate cancer cell lines, EPLIN expression was significantly reduced compared to primary prostate epithelial cells (PrEC), whereas the prostate specific antigen (PSA) positive LNCaP and LAPC4 prostate cancer cell lines failed to express EPLIN $\alpha$ at all [4]. This notion of EPLIN loss is also seen in breast cancer where EPLIN expression in cell lines BT-20, SKBr-3, MCF-7, T-47D and MDA-MB-231 was either reduced or completely lost [4]. Lastly, the authors demonstrated EPLIN as a putative tumour suppressor molecule, where overexpression of EPLIN $\alpha$ caused a reduction in cancer cell growth [4]. Interestingly, when EPLIN $\alpha$ was depleted in breast cancer cell lines, EPLIN $\beta$ either remained consistent or actually increased [4]. This illustrates the potential cancer protective effects that the EPLIN $\alpha$ isoform may exert in various cancer cell systems. EPLIN overexpression has also proved effective in altering the growth phenotype and morphology in additional cell systems including anchorage-independent NIH3T3 transformed cells [7]. Using a soft agar assay and utilising the activated $\mathrm{Cdc} 42$ or the chimeric nuclear oncogene EWS/Fli-1 to transform NIH3T3 cells, EPLIN overexpression resulted in a $\sim 80 \%$ decrease in colony formation for $\mathrm{Cdc} 42$ transformed cells, with a similar growth decrease in EWS/Fli-1 transformed cells [7]. Interestingly, EPLIN displayed heterogeneous staining throughout Ras cells rather than localisation to the actin cytoskeleton [7]. This implies that oncogenic transformation affects the EPLIN/actin architecture, and thus, the localisation of EPLIN to the actin cytoskeleton may be important to exert its suppressive ability [7].

\subsection{Prostate cancer}

There is increasing evidence to suggest that EPLIN is implicated in the development of prostate cancer and the process of 


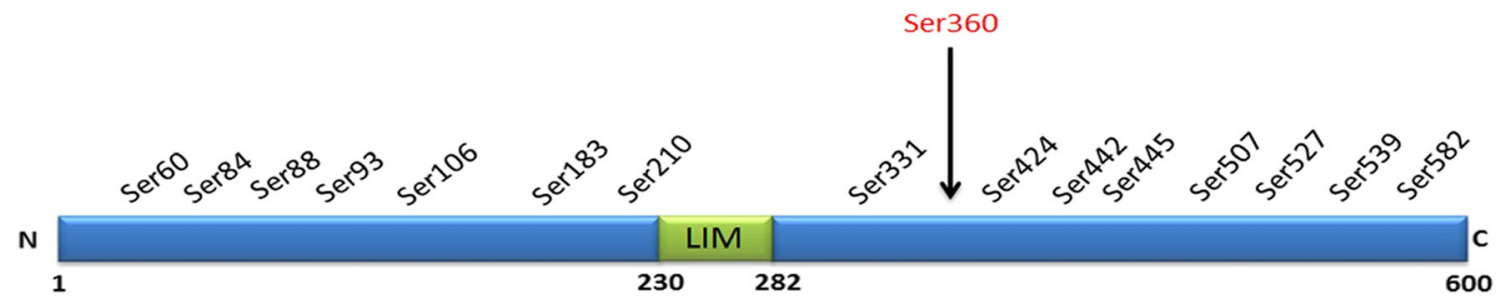

Fig. 6 Predicted phosphorylation sites in EPLIN $\alpha$. The protein structure of human EPLIN has multiple putative phosphorylation sites at all regions of the protein, including various sites where serine kinases

EMT. EMT is a process where polarised epithelial cells are downregulated and subjected to biochemical and morphological changes. Epithelial cells can become transformed to a mesenchymal cell phenotype, losing their cell polarity and cell adhesion at cellular junctions [45]. The converted mesenchymal cell phenotype has a reorganised cytoskeleton and experiences alterations in cell signalling which engenders enhanced migratory and invasiveness capabilities and increased resistance to apoptosis $[45,46]$. During these cellular changes, the actin molecular architecture is disrupted and protein complexes like the cadherin-catenin complex and epithelial

\section{PROSTATE}

(a)

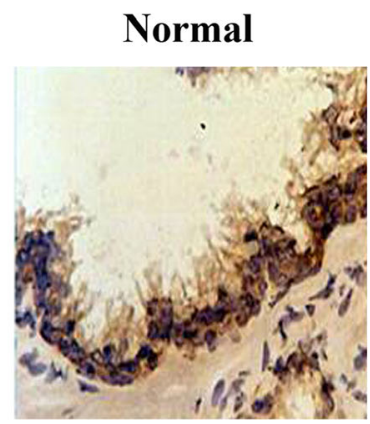

(c)

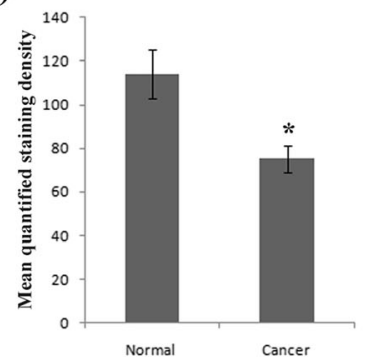

Cancer

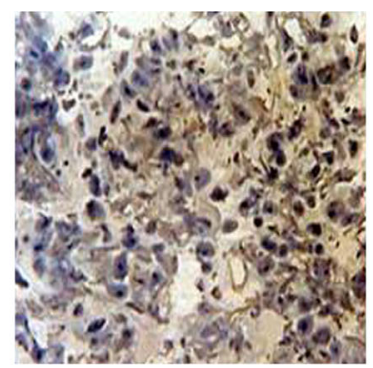

(d)

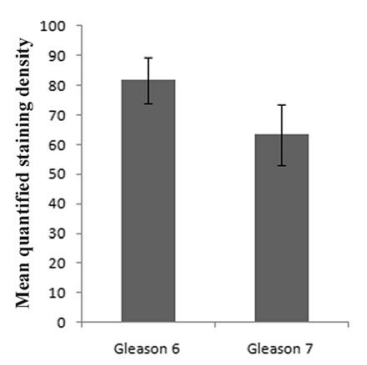

would likely act. Predicted threonine and tyrosine phosphorylation sites not shown. The phosphorylated residue suggested in [20] is indicated. Phosphorylation status predicted using NetPhos 2.0 software

markers are lost [47]. EPLIN is associated with the cadherin-catenin complex and contributes to functional cytoskeletal dynamics [17]. Zhang and co-workers [47] used biochemical and functional approaches to demonstrate that EPLIN is a negative regulator of EMT and invasiveness in prostate cancer (PCa) cells. EPLIN was significantly decreased in cells of more mesenchymal morphology (known as the androgen refractory cancer of the prostate (ARCaPM) cell lineage model), suggesting that EPLIN downregulation is directly implicated in EMT, along with the cadherin-catenin complex [47]. Depletion of EPLIN also provokes various

\section{BREAST}

(b)

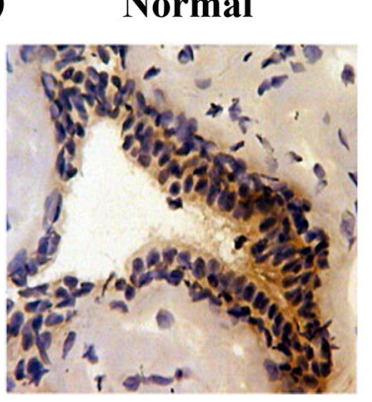

(e)

(f)
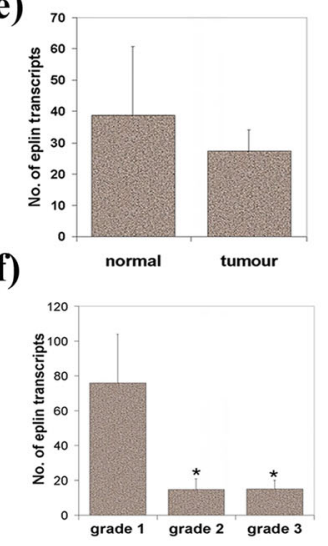

Cancer

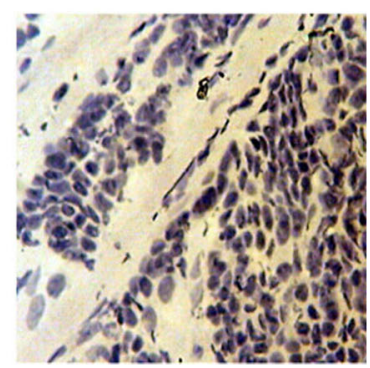

(g)

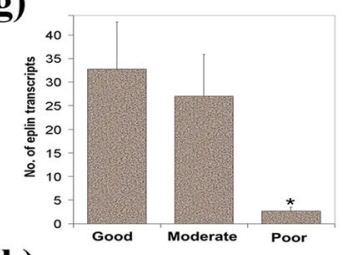

(h)

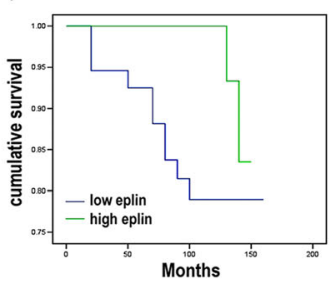

Fig. 7 EPLIN profile in clinical prostate and breast cancer. Immunohistochemical staining $(\times 20$ objective magnification $)$ of normal and cancerous a prostate and $\mathbf{b}$ breast clinical samples demonstrating EPLIN localisation and expressional differences. c, d Semi-quantitative analysis of EPLIN staining within prostate clinical cohort demonstrates that lower levels of EPLIN staining are associated with cancerous and higher-grade samples. e Within a clinical breast cancer cohort, lower transcript expression of EPLIN is seen in tumour samples compared to normal breast tissue and was associated with a higher grade (f), a poorer patient prognosis (g) and reduced overall survival rates (h). Figure modified from $[10,12]$ 
Table 2 Summary of EPLIN clinical implications

\begin{tabular}{|c|c|c|}
\hline $\begin{array}{l}\text { Clinical } \\
\text { implication }\end{array}$ & Description & Reference \\
\hline Prostate cancer & $\begin{array}{l}\text { (1) Immunoblot analyses demonstrated that EPLIN expression in prostate cancer cell lines and xenograft tumours is } \\
\text { reduced compared to prostate epithelial cells (PcEC). PC-3, DU-145, LNCaP, LAPC4, LAPC3 and LAPC9 all } \\
\text { displayed loss of EPLIN protein. } \\
\text { (2) IHC analyses of normal and cancerous clinical prostate sections revealed a greatly reduced staining pattern of } \\
\text { EPLIN in tumour samples. Overexpression of EPLIN in PC-3 cells negatively impacted cell growth in vitro and } \\
\text { in vivo, and were less invasive and had reduced adhesion to the ECM. } \\
\text { (3) EPLIN is implicated in the process of EMT, and IHC analysis revealed that EPLIN loss is correlated with prostate } \\
\text { cancer progression, with a significant reduction of EPLIN expression in tissues with lymph node metastases } \\
\text { compared to primary tumours and normal prostate tissues. }\end{array}$ & $\begin{array}{l}(1)[4] \\
\text { (2) }[12] \\
\text { (3) }[10 \\
\quad 47]\end{array}$ \\
\hline Breast cancer & $\begin{array}{l}\text { (1) Immunoblot analysis revealed reduced or abolished expression of EPLIN protein in tumourigenic breast cancer } \\
\text { cell lines (BT-20, SK-Br-3, MCF-7, T-47D and MDA-MB-231) compared to mammary epithelial cells (MEC), } \\
\text { immortalised mammary epithelial cells (IMEC) and a non-tumourigenic breast cancer cell line, HBL-100. } \\
\text { (2) Analysis by Q-PCR revealed lower levels of EPLIN } \alpha \text { in tumour samples compared to normal. Higher-tumour- } \\
\text { grade samples had lower EPLIN transcript. Patients with poorer prognosis and patients who died of the cancer had } \\
\text { significantly lower levels of EPLIN transcript. Overexpression of EPLIN rendered cells less invasive, and had a } \\
\text { reduced growth rate in vitro and in vivo and were less motile. } \\
\text { (3) IHC analyses displayed a reduction in EPLIN staining in tissues of breast cancer lymph node metastases compared } \\
\text { to primary breast tumours. }\end{array}$ & $\begin{array}{l}\text { (1) }[4] \\
\text { (2) }[10] \\
\text { (3) }[47]\end{array}$ \\
\hline $\begin{array}{l}\text { Oesophageal } \\
\text { cancer }\end{array}$ & $\begin{array}{l}\text { Q-PCR analyses revealed lower levels of EPLIN } \alpha \text { transcript in tumour tissues compared to normal. Higher-tumour- } \\
\text { grade samples had lower EPLIN transcript. Patients who died of the cancer had significantly lower levels of EPLIN } \\
\text { transcript. Patients with local advanced T stage cancer (T2-T4) and patients with lymphatic metastasis had lower } \\
\text { levels of EPLIN } \alpha \text { transcript. Overexpression analysis caused cells to be less invasive and to have a reduced growth rate } \\
\text { in vitro and in vivo. }\end{array}$ & {$[11]$} \\
\hline $\begin{array}{l}\text { Pulmonary } \\
\text { cancer }\end{array}$ & $\begin{array}{l}\text { Q-PCR analyses revealed reduced levels of EPLIN } \alpha \text { transcript in tumour samples compared to normal. Tissues of a } \\
\text { higher TNM stage and where there was nodal involvement also had lower EPLIN transcript. Overexpression } \\
\text { analysis revealed a reduction of cell growth and motility in the SKMES-1 cell line. }\end{array}$ & [14] \\
\hline $\begin{array}{l}\text { Colorectal } \\
\text { cancer }\end{array}$ & $\begin{array}{l}\text { IHC analyses revealed that EPLIN is significantly reduced in lymph node metastatic tumours compared to primary } \\
\text { tumours in colorectal cancer. }\end{array}$ & {$[47]$} \\
\hline $\mathrm{SCCHN}$ & $\begin{array}{l}\text { IHC analyses revealed a reduction of EPLIN staining of cancerous tissue with lymph node metastasis compared to } \\
\text { primary tumours. }\end{array}$ & [47] \\
\hline Oral cancer & $\begin{array}{l}\text { Northern analyses determined that EPLIN expression in } 8 / 8 \text { oral cancer cell lines is reduced compared to control } \\
\text { G3PDH. }\end{array}$ & [4] \\
\hline
\end{tabular}

other morphological changes including disassembly of AJ, increased migratory and invasive potential of cells in vitro, activation of $\beta$-catenin signalling, increased expression of vimentin, increased chemoresistance and decreased expression and nuclear translocation of E-cadherin [47]. Lastly, the authors used immunohistochemistry to show that EPLIN downregulation is correlated with cancer progression in multiple cancer models including lymph node metastasis in PCa, where EPLIN expression was significantly reduced [47]. Altogether, this illustrates that EPLIN may be involved in the regulation of EMT and PCa progression and loss of EPLIN can lead to diverse downstream cellular effects. EPLIN in PCa has also been recently evaluated by our laboratory using the classical PCa cell line, PC-3. By immunohistochemistry (IHC), EPLIN displayed a significant decrease in staining for tumour cells in comparison to normal (see Fig. 7a) [12]. This is accompanied by quantification of staining intensity within the cohort, where lower levels of EPLIN staining are associated with cancerous and higher-tumour-grade samples (see Fig. 7c, d) [12]. Overexpression analysis of EPLIN $\alpha$ resulted in decreased growth rate in tumour cells in vitro, along with reduced invasiveness and cell adhesion to the extracellular matrix (ECM) [12]. Mice injected with PC-3 cells overexpressing EPLIN $\alpha$ developed tumours at a markedly decreased rate in comparison to control [12]. Furthermore, cells overexpressing EPLIN $\alpha$ displayed a greater staining pattern for the focal adhesion targeting protein, paxillin, implying that EPLIN may also be present at these plaques [4, 12]. Altogether, these results demonstrate the potential of EPLIN for monitoring PCa progression and how it can be manipulated to suppress PCa via impeding cancerous traits.

\subsection{Breast cancer}

Our lab has recently evaluated EPLIN involvement in cancer progression in a number of model systems [10-14]. By comparing EPLIN $\alpha$ IHC staining in normal $v s$ tumour cells in breast cancer progression, EPLIN $\alpha$ was found to be 


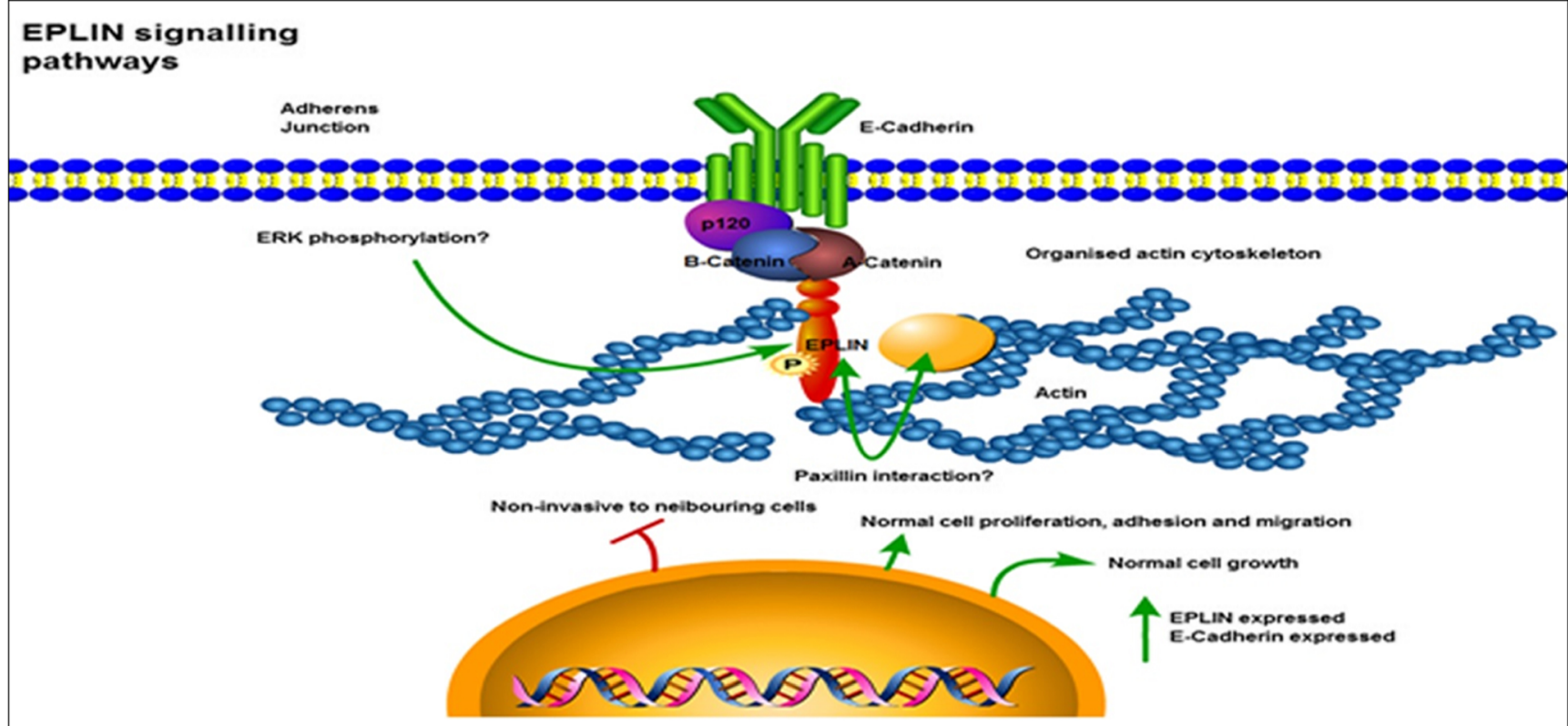

\section{EPLIN loss in cancer: signalling pathways}

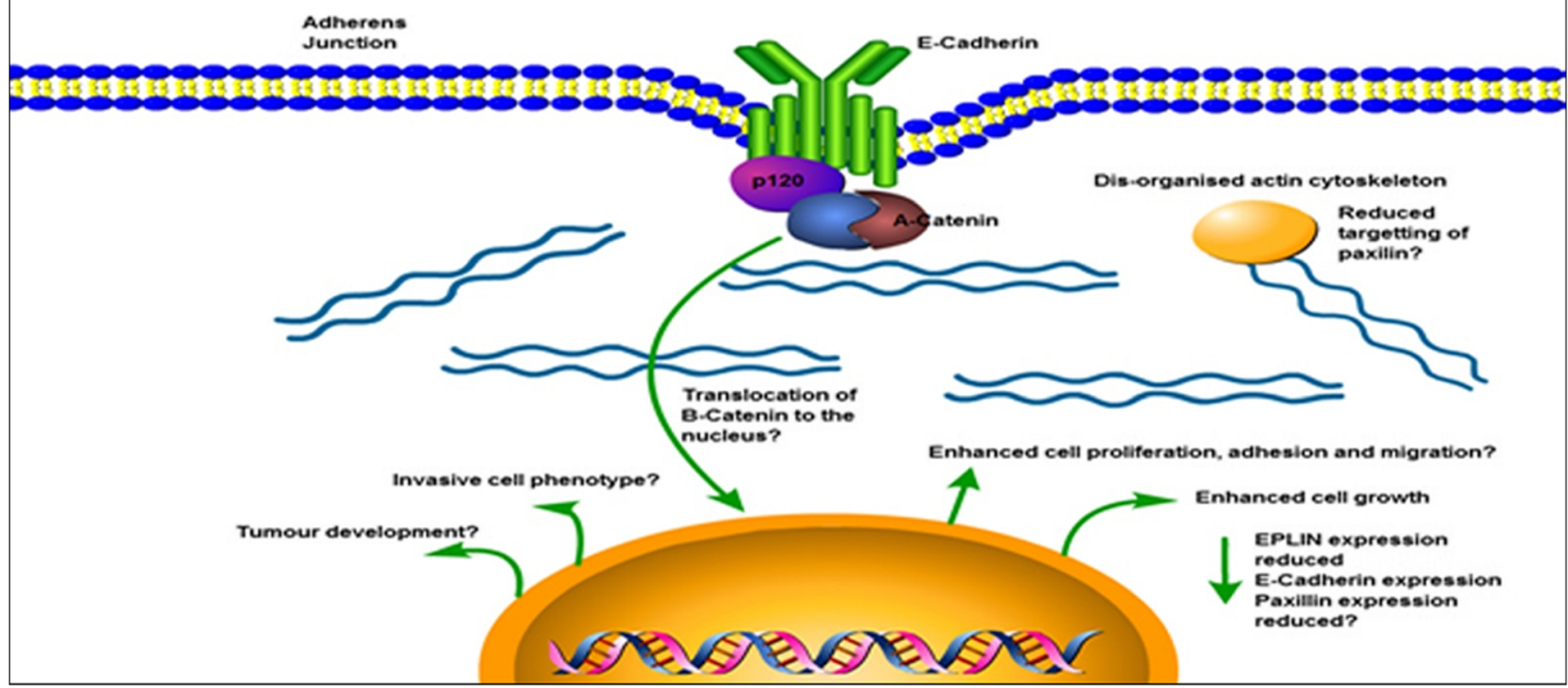

Fig. 8 Proposed EPLIN signalling pathways and implications for loss in cancer. When cancer is not present, EPLIN associates with the actin cytoskeleton linking the cadherin-catenin complex to F-actin via interaction with $\alpha$-catenin. The signal transduction protein, paxillin, interacts with EPLIN in the cytoplasm, and this complex likely stabilises actin dynamics. ERK phosphorylates EPLIN regulating cell motility and migration. When cancer is present and EPLIN is lost, the actin cytoskeleton becomes less organised and this induces membrane ruffling. Paxillin targeting is likely lost reducing focal adhesion between the cadherincatenin complex and actin. These molecular, cellular and morphological consequences may result in increased metastatic potential including enhanced cell migration and motility. Signalling pathways summarised from [12, 16, 20, 47]. Image generated using Pathway Builder 2.0 software substantially weaker in tumour cells than in normal epithelial cells (see Fig. 7b) [10]. This correlated with lower EPLIN $\alpha$ transcript in tumour samples compared to normal samples (see Fig. 7e) with lower EPLIN levels being associated with higher tumour grade, a poorer patient prognosis and reduced overall survival rates (see Fig. $7 \mathrm{f}-\mathrm{h}$ ) [10]. IHC analyses in breast cancer from additional research groups also show EPLIN loss as the tumour becomes more aggressive, specifically comparing EPLIN immunointensity of primary tumours vs tumours with lymph node metastases [47]. Lastly, in vitro and in vivo overexpression analysis of EPLIN highlighted significant reductions in cell growth and cell invasion using transfected 
breast cancer cell lines, and also, highly significant reductions in tumour size were observed in nude mice inoculated with EPLIN- $\alpha$-transfected $v s$ control MDA-MB-231 breast cancer cells [10].

\subsection{Further pathological implications}

Clinical implications for EPLIN also include oesophageal and pulmonary cancer (Table 2). Q-PCR analysis displayed reduced expression of EPLIN $\alpha$ in an oesophageal cancer cohort for both cancerous tissue and cancer cell models [11]. With regard to tumour histological grade, tumour-node-metastasis (TNM) status, nodal status and survival status, EPLIN $\alpha$ transcript generally decreased with levels significantly lower in patients who ultimately died from the cancer, suggesting that EPLIN $\alpha$ is implicated in oesophageal cancer progression and may give an indication for cancer prognosis [11]. Overexpression analysis of EPLIN $\alpha$ in the KYSE150 cell line resulted in decreased growth and invasiveness compared to normal, suggesting that EPLIN $\alpha$ has tumour-suppressive ability by regulating cellular aggressiveness in oesophageal cancer [11]. In a pulmonary cancer cohort also conducted by our lab, Q-PCR analysis showed a reduction of EPLIN $\alpha$ expression in tumour $v s$ normal samples, where EPLIN was also reduced in later TNM stages and cancers with lymph node involvement [14]. Using the SKMES- 1 cell line, overexpression of EPLIN $\alpha$ via transfection in the pEF6 expression vector inhibited cell growth and cell motility [14]. In addition to the apparent molecular loss of EPLIN in various cancers, EPLIN also appears to be reduced at the protein level for colorectal cancer and squamous cell carcinoma of the head and neck ( $\mathrm{SCCHN}$ ), where IHC analysis revealed that EPLIN is decreased in cancers with lymph node metastases $v s$ primary tumours [47].

Collectively, these studies suggest EPLIN may be a clinical indicator for cancer progression in addition to providing further evidence of a tumour-suppressive role for EPLIN $\alpha$ in the regulation of cancer progression.

Finally, in addition to the implication of EPLIN in the spread and progression of cancer, a recent publication provides a link between EPLIN and renal diseases where patients with either membranoproliferative glomerulonephritis (MPGN) or IgA nephropathy had a decreased expression profile for EPLIN via IHC analysis [16]. This advocates the idea that EPLIN may be involved in the pathology of various disease states.

\subsection{Angiogenesis}

Angiogenesis is the formation of new blood vessels from preexisting vessels and is essential for wound healing and normal growth and development. The angiogenic process is frequently utilised by cancer cells, by a means of metastasis, to reach secondary sites around the body and develop secondary tumours. Angiogenesis is therefore a critical factor when targeting cancer therapies. EPLIN $\alpha$ demonstrates a suppressive role in angiogenesis, where overexpression analysis in the HECV endothelial cell line resulted in a reduced capacity to generate tubular structures in a Matrigel tubule formation assay when compared to vector controls [13]. This regulatory effect was also apparent in vivo where mice injected with HECV cells overexpressing EPLIN $\alpha$ in conjunction with cancer cells developed tumours significantly slower than controls [13]. Forced expression also appears to exert an effect on cell matrix adhesion and migration capabilities in this cell line where cells overexpressing EPLIN $\alpha$ both migrated at a significantly slower rate and were significantly less able to adhere to the Matrigel basement membrane [13]. This suppressive role in angiogenesis illustrates that EPLIN $\alpha$ has potentially various regulatory mechanisms for reducing cancer metastasis and could be an effective target for cancer therapy.

\section{Conclusions and outlook}

The interaction of EPLIN and actin has provided an excellent model for investigating multiple aspects of cancer progression over the last decade. The discovery of EPLIN led to a subtle paradigm shift in structural view and organisation of cytoskeletal dynamics, with the acknowledgment that various actinrelated molecules contribute to multiple dynamic processes underlying cellular migration and invasion. There is an established link between EPLIN and cancer progression with frequent downregulation of EPLIN in more aggressive cell lines, reduced staining in cancerous tissue samples and reduced growth potentials when there is forced expression of the EPLIN $\alpha$ isoform in vitro and in vivo. EPLIN is functionally linked to molecules like actin and paxillin and has been implicated in a number of potential pathways to enhance metastatic potential (outlined in Fig. 8). However, the precise mechanistic action of EPLIN and, subsequently, how EPLIN loss contributes to the development of cancer remain elusive. Mechanistic investigations will therefore be crucial to elucidate the full importance of EPLIN in cancer pathophysiology.

Acknowledgments The authors are grateful to Cancer Research Wales and the Welsh Life Science Network - Ser Cymru for supporting this work.

Conflict of interest The authors declare that they have no conflict of interest.

Open Access This article is distributed under the terms of the Creative Commons Attribution 4.0 International License (http:// creativecommons.org/licenses/by/4.0/), which permits unrestricted use, distribution, and reproduction in any medium, provided you give appropriate credit to the original author(s) and the source, provide a link to the Creative Commons license, and indicate if changes were made. 


\section{References}

1. Stewart, B., \& Wild, C. (2014). World Cancer Report 2014.

2. CancerResearchUK (2015). Cancer stats: cancer statistics for the UK. http://www.cancerresearchuk.org/cancer-info/cancerstats/. Accessed 20th November 2014.

3. Chang, D. D., Park, N. H., Denny, C. T., Nelson, S. F., \& Pe, M. (1998). Characterization of transformation related genes in oral cancer cells. Oncogene, 16(15), 1921-1930. doi:10.1038/sj.onc. 1201715.

4. Maul, R. S., \& Chang, D. D. (1999). EPLIN, epithelial protein lost in neoplasm. Oncogene, 18(54), 7838-7841. doi:10.1038/sj.onc. 1203206.

5. Chen, S., Maul, R. S., Kim, H. R., \& Chang, D. D. (2000). Characterization of the human EPLIN (Epithelial Protein Lost in Neoplasm) gene reveals distinct promoters for the two EPLIN isoforms. Gene, 248(1-2), 69-76.

6. Brown, M. C., Perrotta, J. A., \& Turner, C. E. (1996). Identification of LIM3 as the principal determinant of paxillin focal adhesion localization and characterization of a novel motif on paxillin directing vinculin and focal adhesion kinase binding. Journal of Cell Biology, 135(4), 1109-1123.

7. Song, Y., Maul, R. S., Gerbin, C. S., \& Chang, D. D. (2002). Inhibition of anchorage-independent growth of transformed NIH3T3 cells by epithelial protein lost in neoplasm (EPLIN) requires localization of EPLIN to actin cytoskeleton. Molecular Biology of the Cell, 13(4), 1408-1416. doi:10. 1091/mbc.01-08-0414.

8. Maul, R. S., Sachi Gerbin, C., \& Chang, D. D. (2001). Characterization of mouse epithelial protein lost in neoplasm (EPLIN) and comparison of mammalian and zebrafish EPLIN. Gene, 262(1-2), 155-160.

9. Wang, H., Wang, H., Zhu, Z., Yang, S., Feng, S., \& Li, K. (2007). Characterization of porcine EPLIN gene revealed distinct expression patterns for the two isoforms. Animal Biotechnology, 18(2), 101-108. doi:10.1080/10495390600864660.

10. Jiang, W. G., Martin, T. A., Lewis-Russell, J. M., Douglas-Jones, A., Ye, L., \& Mansel, R. E. (2008). Eplin-alpha expression in human breast cancer, the impact on cellular migration and clinical outcome. Molecular Cancer, 7, 71. doi:10.1186/1476-4598-7-71.

11. Liu, Y., Sanders, A. J., Zhang, L., \& Jiang, W. G. (2012). EPLINalpha expression in human oesophageal cancer and its impact on cellular aggressiveness and clinical outcome. Anticancer Research, 32(4), 1283-1289.

12. Sanders, A. J., Martin, T. A., Ye, L., Mason, M. D., \& Jiang, W. G. (2011). EPLIN is a negative regulator of prostate cancer growth and invasion. Journal of Urology, 186(1), 295-301. doi:10.1016/j.juro. 2011.03.038

13. Sanders, A. J., Ye, L., Mason, M. D., \& Jiang, W. G. (2010). The impact of EPLINalpha (Epithelial protein lost in neoplasm) on endothelial cells, angiogenesis and tumorigenesis. Angiogenesis, 13(4), 317-326. doi:10.1007/s10456-010-9188-7.

14. Liu, Y., Sanders, A. J., Zhang, L., \& Jiang, W. G. (2012). Expression profile of Epithelial Protein Lost in Neoplasm-Alpha $($ EPLIN- $\alpha)$ in human pulmonary cancer and its impact on SKMES1 cells in vitro. Journal of Cancer Therapy, 3, 452-459. doi:10. 4236/jct.2012.324058.

15. Maul, R. S., Song, Y., Amann, K. J., Gerbin, S. C., Pollard, T. D., \& Chang, D. D. (2003). EPLIN regulates actin dynamics by crosslinking and stabilizing filaments. Journal of Cell Biology, 160(3), 399-407. doi:10.1083/jcb.200212057.

16. Tsurumi, H., Harita, Y., Kurihara, H., Kosako, H., Hayashi, K., Matsunaga, A., et al. (2014). Epithelial protein lost in neoplasm modulates platelet-derived growth factor-mediated adhesion and motility of mesangial cells. Kidney International, 86(3), 548-557. doi:10.1038/ki.2014.85.

17. Abe, K., \& Takeichi, M. (2008). EPLIN mediates linkage of the cadherin catenin complex to F-actin and stabilizes the circumferential actin belt. Proceedings of the National Academy of Sciences of the United States of America, 105(1), 13-19. doi:10.1073/pnas. 0710504105.

18. Smith, T. C., Fang, Z., \& Luna, E. J. (2010). Novel interactors and a role for supervillin in early cytokinesis. Cytoskeleton (Hoboken), 67(6), 346-364. doi:10.1002/cm.20449.

19. Karakose, E., Geiger, T., Flynn, K., Lorenz-Baath, K., Zent, R., Mann, M., et al. (2015). The focal adhesion protein PINCH-1 associates with EPLIN at integrin adhesion sites. Journal of Cell Science, 128(5), 1023-1033. doi:10.1242/jcs.162545.

20. Han, M. Y., Kosako, H., Watanabe, T., \& Hattori, S. (2007). Extracellular signal-regulated kinase/mitogen-activated protein kinase regulates actin organization and cell motility by phosphorylating the actin cross-linking protein EPLIN. Molecular and Cellular Biology, 27(23), 8190-8204. doi:10.1128/mcb.00661-07.

21. Steder, M., Alla, V., Meier, C., Spitschak, A., Pahnke, J., Furst, K., et al. (2013). DNp73 exerts function in metastasis initiation by disconnecting the inhibitory role of EPLIN on IGF1R-AKT/ STAT3 signaling. Cancer Cell, 24(4), 512-527. doi:10.1016/j.ccr. 2013.08.023.

22. Seong, B. K., Lau, J., Adderley, T., Kee, L., Chaukos, D., Pienkowska, M., et al. (2014). SATB2 enhances migration and invasion in osteosarcoma by regulating genes involved in cytoskeletal organization. Oncogene. doi:10.1038/onc.2014.289.

23. Ohoka, A., Kajita, M., Ikenouchi, J., Yako, Y., Kitamoto, S., Kon, S., et al. (2015). EPLIN is a crucial regulator for extrusion of RasV12-transformed cells. Journal of Cell Science, 128(4), 781789. doi:10.1242/jcs.163113.

24. Stricker, J., Falzone, T., \& Gardel, M. L. (2010). Mechanics of the F-actin cytoskeleton. Journal of Biomechanics, 43(1), 9-14. doi:10. 1016/j.jbiomech.2009.09.003.

25. Leitner, L., Shaposhnikov, D., Descot, A., Hoffmann, R., \& Posern, G. (2010). Epithelial Protein Lost in Neoplasm alpha (Eplin-alpha) is transcriptionally regulated by G-actin and MAL/MRTF coactivators. Molecular Cancer, 9, 60. doi:10.1186/1476-4598-960.

26. Kim, J. S., Xu, X., Li, H., Solomon, D., Lane, W. S., Jin, T., et al. (2011). Mechanistic analysis of a DNA damage-induced, PTENdependent size checkpoint in human cells. Molecular and Cellular Biology, 31(13), 2756-2771. doi:10.1128/mcb.01323-10.

27. Ratheesh, A., \& Yap, A. S. (2012). A bigger picture: classical cadherins and the dynamic actin cytoskeleton. Nature Reviews Molecular Cell Biology, 13(10), 673-679. doi:10.1038/nrm3431.

28. Meng, W., \& Takeichi, M. (2009). Adherens junction: molecular architecture and regulation. Cold Spring Harbor Perspectives in Biology, 1(6), a002899. doi:10.1101/cshperspect.a002899.

29. Mege, R. M., Gavard, J., \& Lambert, M. (2006). Regulation of cellcell junctions by the cytoskeleton. Current Opinion in Cell Biology, 18(5), 541-548. doi:10.1016/j.ceb.2006.08.004.

30. Yonemura, S. (2011). Cadherin-actin interactions at adherens junctions. Current Opinion in Cell Biology, 23(5), 515-522. doi:10. 1016/j.ceb.2011.07.001

31. Nelson, W. J. (2008). Regulation of cell-cell adhesion by the cadherin-catenin complex. Biochemical Society Transactions, 36(Pt 2), 149-155. doi:10.1042/bst0360149.

32. Drees, F., Pokutta, S., Yamada, S., Nelson, W. J., \& Weis, W. I. (2005). Alpha-catenin is a molecular switch that binds E-cadherinbeta-catenin and regulates actin-filament assembly. Cell, 123(5), 903-915. doi:10.1016/j.cell.2005.09.021.

33. Yamada, S., Pokutta, S., Drees, F., Weis, W. I., \& Nelson, W. J. (2005). Deconstructing the cadherin-catenin-actin complex. Cell, 123(5), 889-901. doi:10.1016/j.cell.2005.09.020. 
34. Cavey, M., Rauzi, M., Lenne, P. F., \& Lecuit, T. (2008). A twotiered mechanism for stabilization and immobilization of Ecadherin. Nature, 453(7196), 751-756. doi:10.1038/nature06953.

35. Taguchi, K., Ishiuchi, T., \& Takeichi, M. (2011). Mechanosensitive EPLIN-dependent remodeling of adherens junctions regulates epithelial reshaping. Journal of Cell Biology, 194(4), 643-656. doi:10. 1083/jcb.201104124.

36. Geiger, B., Tokuyasu, K. T., Dutton, A. H., \& Singer, S. J. (1980). Vinculin, an intracellular protein localized at specialized sites where microfilament bundles terminate at cell membranes. Proceedings of the National Academy of Sciences of the United States of America, 77(7), 4127-4131.

37. Sawyer, J. K., Harris, N. J., Slep, K. C., Gaul, U., \& Peifer, M. (2009). The Drosophila afadin homologue Canoe regulates linkage of the actin cytoskeleton to adherens junctions during apical constriction. Journal of Cell Biology, 186(1), 57-73. doi:10.1083/jcb. 200904001.

38. Chervin-Petinot, A., Courcon, M., Almagro, S., Nicolas, A., Grichine, A., Grunwald, D., et al. (2012). Epithelial protein lost in neoplasm (EPLIN) interacts with alpha-catenin and actin filaments in endothelial cells and stabilizes vascular capillary network in vitro. Journal of Biological Chemistry, 287(10), 7556-7572. doi:10.1074/jbc.M111.328682.

39. Gulino-Debrac, D. (2013). Mechanotransduction at the basis of endothelial barrier function. Tissue Barriers, 1(2), e24180. doi:10. 4161/tisb. 24180 .

40. Heng, Y. W., \& Koh, C. G. (2010). Actin cytoskeleton dynamics and the cell division cycle. International Journal of Biochemistry and Cell Biology, 42(10), 1622-1633. doi:10.1016/j.biocel.2010. 04.007 .
41. Chircop, M., Oakes, V., Graham, M. E., Ma, M. P., Smith, C. M., Robinson, P. J., et al. (2009). The actin-binding and bundling protein, EPLIN, is required for cytokinesis. Cell Cycle, 8(5), 757-764.

42. Pribic, J., \& Brazill, D. (2012). Paxillin phosphorylation and complexing with Erk and FAK are regulated by PLD activity in MDA-MB-231 cells. Cellular Signalling, 24(8), 1531-1540. doi: 10.1016/j.cellsig.2012.03.015.

43. Pearson, L. L., Castle, B. E., \& Kehry, M. R. (2001). CD40mediated signaling in monocytic cells: up-regulation of tumor necrosis factor receptor-associated factor mRNAs and activation of mitogen-activated protein kinase signaling pathways. International Immunology, 13(3), 273-283.

44. Zhang, S., Wang, X., Iqbal, S., Wang, Y., Osunkoya, A. O., Chen, Z., et al. (2013). Epidermal growth factor promotes protein degradation of epithelial protein lost in neoplasm (EPLIN), a putative metastasis suppressor, during epithelial-mesenchymal transition. Journal of Biological Chemistry, 288(3), 1469-1479. doi:10. 1074/jbc.M112.438341.

45. Kalluri, R., \& Weinberg, R. A. (2009). The basics of epithelialmesenchymal transition. Journal of Clinical Investigation, 119(6), 1420-1428. doi:10.1172/jci39104.

46. Lamouille, S., Xu, J., \& Derynck, R. (2014). Molecular mechanisms of epithelial-mesenchymal transition. Nature Reviews Molecular Cell Biology, 15(3), 178-196. doi:10.1038/nrm3758.

47. Zhang, S., Wang, X., Osunkoya, A. O., Iqbal, S., Wang, Y., Chen, Z., et al. (2011). EPLIN downregulation promotes epithelialmesenchymal transition in prostate cancer cells and correlates with clinical lymph node metastasis. Oncogene, 30(50), 4941-4952. doi: 10.1038/onc.2011.199. 